\title{
Propuesta Tecnológica para el Mejoramiento de la Educación y la Inclusión Social en los Niños Sordos
}

\author{
Cesar Hernández ${ }^{\star}$, Hans Márquez, Fernando Martínez \\ Universidad Distrital Francisco José de Caldas, Facultad Tecnológica, Transversal 70 B No. 73 a 35 Sur. \\ Bogotá, Colombia (e-mail: cahernandezs@udistrital.edu.co, hans_marquez@hotmail.com, \\ fmartinezs@udistrital.edu.co) \\ * autor a quien debe ser dirigida la correspondencia
}

Recibido Abr. 14, 2015; Aceptado Jun. 20, 2015; Versión final Ago. 10, 2015, Publicado Dic. 2015

\begin{abstract}
Resumen
El objetivo del presente artículo es presentar una propuesta tecnológica que permita mejorar la inclusión social y la calidad de vida de los niños con discapacidad auditiva. La metodología utilizada se basó en el diseño y desarrollo de un dispositivo electrónico que facilita el aprendizaje autónomo de la lengua de señas colombiana y la comunicación con su entorno. Los principales resultados fueron la reducción en el tiempo de aprendizaje de la lengua de señas, el aumento de autonomía en el proceso de aprendizaje y el mejoramiento de la comunicación con su entorno familiar. Se concluye que la tecnología juega un papel importante y significativo en la reducción de la brecha existente en cuanto a la inclusión social de las personas con discapacidad auditiva.
\end{abstract}

Palabras clave: discapacidad auditiva; aprendizaje; lengua de señas; autónomo, tecnología

\section{Technological Proposal for the Improvement of Education and Social Inclusion in the Deaf Children}

\begin{abstract}
The aim of this paper is to present a technology that improves social inclusion and quality of life of hearing impaired children. The methodology used was based on the design and development of an electronic device that facilitates the autonomous learning of sign language and communication with their environment. The main results were the reduction in learning time sign language, increasing autonomy in the learning process and improving communication with their families. It is concluded that technology plays an important and significant role in reducing the gap in terms of social inclusion of people with hearing impairment.
\end{abstract}

Keywords: deaf impaired; learning; signal speech; autonomous; technology 


\section{INTRODUCCIÓN}

La población con discapacidad auditiva, carece de oportunidades laborales y académicas (Espinosa et al., 2012), como se puede constatar en la encuesta de reconocimiento de la población sorda en Colombia (Instituto Nacional para sordos, 2009), allí se observa que de un total de 99.693 personas con deficiencia auditiva, el $43 \%$ se encuentra en el estrato 1 (clasificación socioeconómica que se realiza en Colombia para definir los impuestos y las tarifas de los servicios públicos) y el $37 \%$ en el estrato 2, mientras que tan solo el $1 \%$ se encuentran en los estratos 4,5 y 6 , lo anterior incide indirectamente en la falta de oportunidades para el ingreso a los estudios universitarios y aún más a los estudios de postgrado, ya que de un total de 98.983 personas que inician estudios de educación superior, solo el $0,255 \%$ han logrado culminar sus estudios de pregrado, mientras que tan solo el $0,0424 \%$ de esta población han terminado estudios de postgrado. Lo anterior no solo un problema en Colombia, sino también a nivel de América Latina (Fuller et al., 2004; Chiroleu, 2011).

Debido a que en América Latina la oferta de herramientas tecnológicas para la población con discapacidad es reducida, y además, costosa por el hecho de ser importadas, hace más difícil a la población sorda poder mejorar su nivel de inclusión social, a través del desarrollo de su potencial en el ámbito laboral y académico, generando una brecha muy acentuada que no permite a las políticas públicas y a los programas de apoyo a la discapacidad, tener significativos avances en esta materia (Alonso y Díaz, 2008; Escalante, 2012). Factores como los comentados con anterioridad, repercuten no solamente en la calidad de vida de la persona sorda sino también en la de sus familiares, al generar dependencia económico-social. La situación descrita se puede presentar incluso desde temprana edad, donde los niños sordos emplean una significativa parte de su tiempo de estudio en aprender la lengua de señas para poder comunicarse efectivamente con sus compañeros, profesores, amigos y familiares. De acuerdo a lo anterior, el presente trabajo ha planteado la siguiente pregunta de investigación: ¿Cómo la tecnología podría facilitar el aprendizaje de la lengua de señas?

Hasta el momento se han realizado varios trabajos investigación que buscan dar solución a la problemática descrita a través de las tecnologías de la información y la comunicación (TIC) (Hernández et al., 2014; Marulanda, et al., 2014). Uno de los primeros trabajos fue el desarrollado en la patente US4074444 por Laenger, et al., 1976), consistente en una mano electromecánica con interfaz de teclado, la cual replicaba el signo correspondiente a la letra digitada por el usuario. Otro trabajo interesante fue la patente US5047952 desarrollada por Kramer, Lindener y George (Kramer et al., 1988), la cual usa un sistema compuesto por un guante que interpreta el movimiento de los dedos mediante galgas de presión dispuestas en las articulaciones, las cuales al percibir un cambio en la presión de estas, envían los datos a un computador el cual mediante reconocimiento de patrones interpreta las señales y las muestra en pantalla. A su vez se puede responder mediante el teclado, a través de un sintetizador de voz, un monitor LCD, o un display Braille.

Actualmente también existen dispositivos que permiten la comunicación entre personas con discapacidad auditiva y personas que pueden oír, como los desarrollados en las patentes FR2960999 de Masfrand y Francois (Masfrand et al., 2010), US2011116608 de Gwendolyn (Gwendolyn, 2011) y CN201984680 de Yang y Zhang (Yang y Zhang, 2011). La persona con discapacidad tiene como interfaz para el envío de mensajes: teclados, lápices ópticos, pantallas táctiles y sistemas de reconocimiento de imágenes, y como interfaz de recepción de mensajes, una pantalla donde se visualizan imágenes de la lengua señas. Por otro lado, la persona sin discapacidad utiliza teclados o micrófonos para enviar información, y altavoces para recibirla.

La patente 4378215 de Sparks (Sparks, 1981) tiene un enfoque hacia el aprendizaje del alfabeto manual, en este trabajo se hace uso de figuras de manos, cada una de ellas formando las señas del alfabeto manual para sordos e incorporado en su base, la representación en alfabeto Braille de la respectiva seña. Por su parte la patente US4368459 de Sapora (Sapora, 1980) muestra un aparato de señalización para coordinar vía visual a estudiantes con discapacidad auditiva, en una ambiente de clases. Los mensajes del profesor son presentados como imágenes o como lengua de señas. El dispositivo debe captar la atención de la persona con discapacidad auditiva con el propósito de que reciba información de su entorno. En el caso de la enseñanza de circuitos analógicos para estudiantes con discapacidad auditiva, la patente CN201845462 de Hao (Hao, 2010) provee una tarjeta de enseñanza, la cual utiliza datos experimentales o imágenes de los experimentos, para explicar los principios de los circuitos analógicos, esta tarjeta se compone de un generador de onda, módulo de energía DC, módulo de amperímetro y una fuente regulada. Finalmente, para la enseñanza a niños con discapacidad auditiva la patente US4799889 de Jockey (Jockey, 1987) hace uso de un oso de peluche que tiene brazos tubulares huecos por los cuales el profesor puede pasar sus brazos y proyectar sus brazos en los brazos del oso de peluche, de esta forma el profesor puede realizar el lenguaje de señas usando un objeto que sea del agrado de los niños. 
En (Rudner et al., 2015) los autores determinan que las habilidades de alfabetización se pueden mejorar mediante la capacitación con el software Omega-is-d1, con un pequeño grupo de estudiantes de una escuela primaria estatal de Suecia para personas sordas y con problemas de audición. Omega-is-d1 es un programa de computador que ha demostrado ser efectivo en el apoyo a los niños durante su desarrollo de lectura. Este software está basado en un modelo pedagógico multimedia e interactivo. Aunque las habilidades de alfabetización fueron en general bajas se demostró que mejoraron sustancialmente y significativamente en el transcurso de la formación.

Los autores del documento "Policy and practice in sign bilingual education: development, challenges and directions" (Swanwick, 2010) proponen tres sugerencias de direcciones futuras para el desarrollo de la investigación y la práctica de signos bilingüe. El primer reto son los modelos sociales y médicos de la sordera, y las implicaciones de estos en la educación, enfatizando la valoración de la sordera, la lengua de signos, la identidad, la autoestima, y reconociendo las características únicas y distintivas de la cultura sorda. El segundo reto que prevalece es el desarrollo de la alfabetización. La lengua de signos enfocada hacia la educación dentro de un ambiente educacional, puede tener ventajas educativas para los estudiantes que son bilingües, debido a que las habilidades aprendidas en un lenguaje pueden ayudar en otro. El tercer reto es el futuro rol de la lengua de signos Británica, debido al rápido cambio del lenguaje y las necesidades de comunicación de los alumnos con sordera profunda.

En (Hernández, et al., 2015) se desarrolló la primera versión del prototipo tecnológico enfocado a facilitar el aprendizaje de la lengua de señas en niños sordos, el cual fue producto de una tesis de pregrado en la Universidad Distrital Francisco José de Caldas. Esta primera versión tuvo varias limitaciones técnicas que fueron mejoradas en la segunda versión (Hernández, 2013) y tercera versión (la que se presenta en este documento). En (Hernández, et al., 2015) se desarrolló un prototipo tecnológico que permitiera interactuar al niño sordo con la Lengua de Señas Colombiana (LSC) a través de una pantalla táctil, sin embargo, a pesar de su tamaño compacto (muy similar al de un celular inteligente de hoy en día) y de la reducción del tiempo en el aprendizaje de la lengua de señas, el dispositivo presentó limitaciones en su autonomía energética, su tamaño de vocabulario, su nivel de interacción con el usuario, la resistencia del material de la carcasa y la diversidad de contenido multimedia. La mayoría de estas limitaciones fueron eliminadas en la segunda versión cuyo resultado tecnológico final fue patentado por la Superintendencia de Industria y Comercio de Colombia. Finalmente en la tercera versión, la cual se describe en este documento, se mejoraron aún más las características limitantes y se resolvieron las que no pudieron mejorar en la segunda versión.

A pesar de las tecnologías desarrolladas y descritas anteriormente, en la actualidad la población con discapacidad auditiva de América Latina y en especial los niños sordos, no cuentan con tecnológicas integrales de bajo costo que permitan mejorar su grado de inclusión social y su nivel de calidad de vida (Hernandez et al., 2015; Chaves et al., 2015). Para dar solución a toda la problemática descrita anteriormente, el presente proyecto de investigación tiene por objetivo desarrollar un dispositivo electrónico, independiente y portable que facilite el aprendizaje autónomo de la lengua de señas colombiana en niños con discapacidad auditiva. Para materializar este objetivo la herramienta tecnológica propuesta se conformó de seis módulos principales: ingreso de datos, visualización, síntesis de voz, reconocimiento, carga de batería y optimización de energía, y un módulo secundario: monitorización del estado de la batería, los cuales se describirán en detalle en la metodología.

La herramienta tecnológica propuesta posee una pantalla grafica táctil que le permite al usuario con discapacidad auditiva, interactuar con todas las aplicaciones desarrolladas sobre el dispositivo, como lo son: aprendizaje del lenguaje de señas, comunicación, reconocimiento de voz, ubicación y entorno. La pantalla grafica táctil permite visualizar los mensajes que sean procesados a través de la unidad de reconocimiento de voz, dispuesta en el dispositivo. De igual forma la pantalla generará un teclado, para que el usuario pueda digitar los mensajes que desea trasmitir, los cuales se reproducirán auditivamente. Para permitir la participación activa de los familiares en el proceso de aprendizaje del lenguaje de señas colombiana por parte del niño, el dispositivo permite la recepción de mensajes de voz y su posterior interpretación, a través de un sistema de reconocimiento independiente del habla, que hace uso de un micrófono tipo capacitivo y un módulo programable para la captura y procesamiento de la información, para visualizar la información en la pantalla. Con respecto a la evaluación y validación del dispositivo se realizaron pruebas de laboratorio en las que se hace una evaluación técnica para asegurar la funcionalidad del mismo y dos pruebas de campo.

\section{LENGUA DE SEÑAS}

La lengua de señas es un tipo de comunicación visual, gestual y no oral, usado por la comunidad con discapacidad auditiva. Esta lengua posee su propia estructura gramatical y es muy diferente de la lengua oral, por tanto, no se da una correspondencia entre la estructura gramatical de ambas lenguas. 
Al respecto Tamarit define la lengua de señas como: «lenguajes naturales que se han desarrollado, al menos en parte, independientemente del lenguaje hablado, y no se corresponden lingüísticamente con él, teniendo sus propias estructuras sintácticas y organizativas bastante flexibles» (Tamarit, 1998).

De la misma forma que existen diferentes lenguajes en el mundo, ocurre con la lengua de señas, las cuales, varían según el país. Tal es el caso de la lengua de signos americana ASL o Ameslan, el Lenguaje de Signos Británico BSL, entre otras comunidades.

En el caso colombiano, la lengua de señas fue reconocida hasta el año 1996, bajo el mandato del Presidente Ernesto Samper Pizano, en la ley 324, artículo 2, el cual reza así: "El estado colombiano reconoce la lengua de señas como propia de la comunidad sorda del país". La característica de esta lengua es que es visual y corporal, ya que hace uso del cuerpo para expresar las palabras y por tanto, la parte visual es un factor importante para reconocer lo que se está tratando de comunicar (Instituto Nacional para Sordos, 2013).

La divulgación, enseñanza y estudio de la lengua de señas y su articulación, se empezó a realizar antes de la proclamación de la ley 324, más exactamente desde 1984. Gracias a dicho trabajo, se fue conformando un grupo de investigación para el "lenguaje manual colombiano", como se llamaba en sus inicios. Resultado de esas primeras aproximaciones y de la enseñanza de la lengua, se crearon unas cartillas por parte de la Federación Nacional de Sordos de Colombia (Fenascol).

\section{Implicaciones Comunicativas}

Un aspecto que se ve afectado cuando una persona ha perdido total o parcialmente la capacidad auditiva, es el mecanismo por el cual se va a comunicar con su familia o personas en su entorno. Cabe destacar que las personas que han perdido la capacidad auditiva usan medios orales o manuales (Lengua de señas) para la comunicación o una combinación de ambos. La comunicación oral incluye lenguaje, lectura labial, y el uso de la capacidad de oído residual en caso de que la persona tenga pérdida parcial de audición (Skliar, 1997; Tur y Fernández, 1992).

En el caso del niño que es sordo y que sus familiares comparten también este tipo de discapacidad, la comunicación entre éste y sus familiares, se realiza a través de lengua de señas, logrando una mayor interacción y participación, a través del conocimiento compartido entre ambos, lo cual es fundamental en su desarrollo. Sin embargo, en el caso del niño sordo con padres oyentes, la interacción entre ambos es complicada, debido a que el niño se encuentra en un entorno en el que habitualmente se utiliza el lenguaje oral, al cual no tiene acceso. Esto ocasiona que el proceso de desarrollo y aprendizaje para el niño sea mucho más complejo, obligando a los familiares a conocer y aprender la lengua de señas, a fin de poder interactuar con el niño.

La comunicación manual o lengua de señas, se refiere a la representación manual de signos y letras del alfabeto. La combinación de la lengua de señas y la lectura labial se conoce como comunicación total.

Las personas con capacidad auditiva residual pueden hacer uso de implementos tecnológicos como implantes cocleares o audífonos, para potencializar esa capacidad residual y permitirles el acceso al lenguaje hablado. Sin embargo, deben estudiarse cuidadosamente estas posibilidades para cada caso en particular, de forma que se ofrezca la mejor alternativa para cada persona según las fortalezas y necesidades de cada una.

\section{Implicaciones Educativas}

Aunque la persona sorda no presenta ningún tipo de incapacidad intelectual o cognitiva, es necesario contar con algunos servicios especiales para garantizar una adecuada educación (Domínguez \& Rodríguez, 2003), como: intérpretes de la lengua de señas, películas y videos subtitulados, localización preferencial en el aula de clases como por ejemplo en la primera fila para facilitar la lectura hablada en la sala de clases, entrenamiento de locución y sistema auditivo por parte de un especialista para aquellas personas que tienen capacidad auditiva residual, y sistemas de amplificación en el aula de clase, entre otras.

Se debe tener en cuenta que los niños sordos tendrán una mayor dificultad a la hora de aprender el vocabulario, expresiones, el orden de las letras en el alfabeto y otros aspectos de la comunicación. Esta dificultad se verá más acentuada en los niños con perdida prelocutiva, que en perdida perilocutiva y poslocutiva, en donde el niño posee las bases del lenguaje y cuenta con un marco de referencia en cuanto a lenguaje corresponde. 
Por la anterior razón, se hace necesario el uso temprano de métodos visibles de comunicación, tales como la lengua de señas, el alfabeto manual, la palabra complementada, y en el caso de niños con capacidad auditiva residual el uso de amplificadores y entrenamiento oral para evitar en lo posible, un atraso en el lenguaje (Salvador y Gutiérrez, 2006).

Los padres y los docentes de la escuela juegan un papel importante en el proceso de aprendizaje, ya que el trabajo conjunto de estos, permitirá un proceso de aprendizaje continuo, en el que se buscará la consecución de metas anuales, con el acompañamiento tanto de docentes como de familiares.

\section{METODOLOGIA}

El diseño del prototipo se orientó a una herramienta de aprendizaje de la lengua de señas de fácil comprensión y manejo. Además, el prototipo debe ser un elemento portable, permitiendo a la persona con discapacidad auditiva llevarlo consigo en todo momento. Para el desarrollo del prototipo se escogió una pantalla grafica táctil, facilitando al máximo la interacción del prototipo con el usuario, y desde la cual el usuario pudiera controlar todas las actividades. Se diseñó una caja que fuese lo más pequeña posible y se trató asimismo que el prototipo, fuera liviano, atendiendo al propósito de portabilidad del mismo. Con estos criterios en mente se diseñó el dispositivo compuesto por los cuatro módulos mostrados en la Fig. 1.

\section{Módulo de Ingreso de datos}

El diseño de este módulo se compone fundamentalmente de una pantalla grafica táctil, por la cual el usuario puede navegar y seleccionar cada uno de los menús disponibles en el prototipo. Una vez el prototipo es encendido, muestra un mensaje de bienvenida, y seguidamente los tres menús disponibles para el usuario (ver Fig. 2): menú de lecciones, menú de comunicación y menú de reconocimiento de voz.

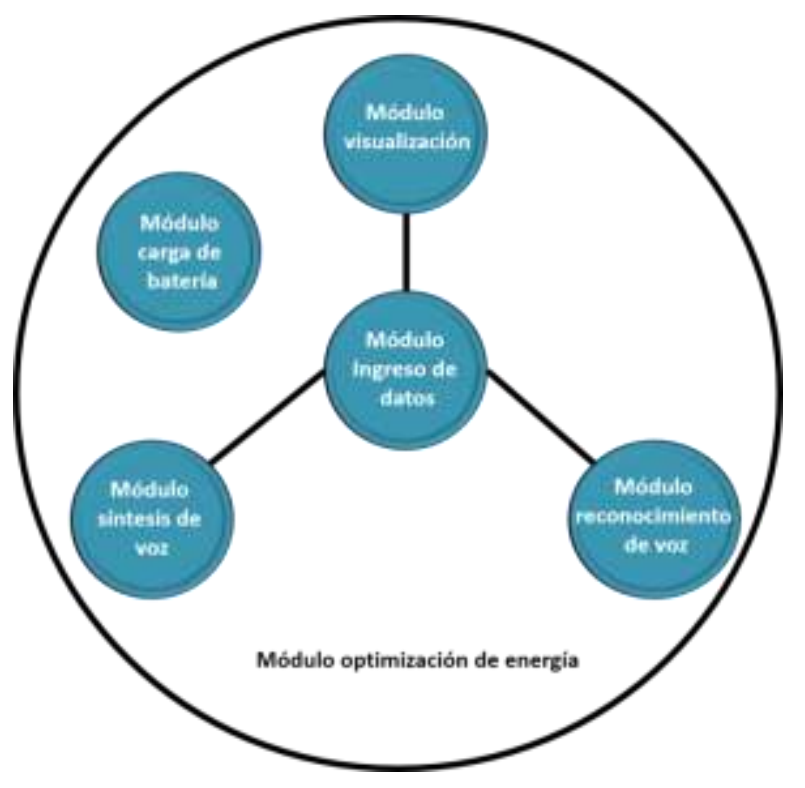

Fig. 1: Esquema en Bloques de módulos que integran el prototipo

Si la opción escogida por el usuario es el menú lecciones, el sistema mostrara las lecciones disponibles en el prototipo, como por ejemplo: letras, números, meses, días, colores y saludos, entre otras. De acuerdo con la lección escogida, el dispositivo mostrará las opciones específicas disponibles para dicha lección, como por ejemplo el alfabeto en el caso de seleccionar la lección letras. Cuando el usuario selecciona alguna letra del alfabeto, el sistema iniciará un video donde se explica la seña que identifica la opción seleccionada, el cual se repetirá hasta que el usuario lo desee.

Cuando el usuario selecciona el menú comunicaciones, el prototipo muestra una nueva pantalla donde habilita un teclado QWERTY virtual, en la parte inferior de la pantalla y habilita un espacio en blanco en la parte superior de la misma, con el objetivo que lo que escriba el usuario, pueda ser visualizado antes de enviar el texto al sintetizador de voz para convertirlo en voz. El teclado virtual de la pantalla táctil incluye las teclas "Borrado", "Espacio" y "Enter", con sus respectivas funciones, en el caso de la tecla "Enter", su función es enviar la frase escrita a través del teclado, hacia el sintetizador de voz para realizar el proceso de conversión de texto a voz. 


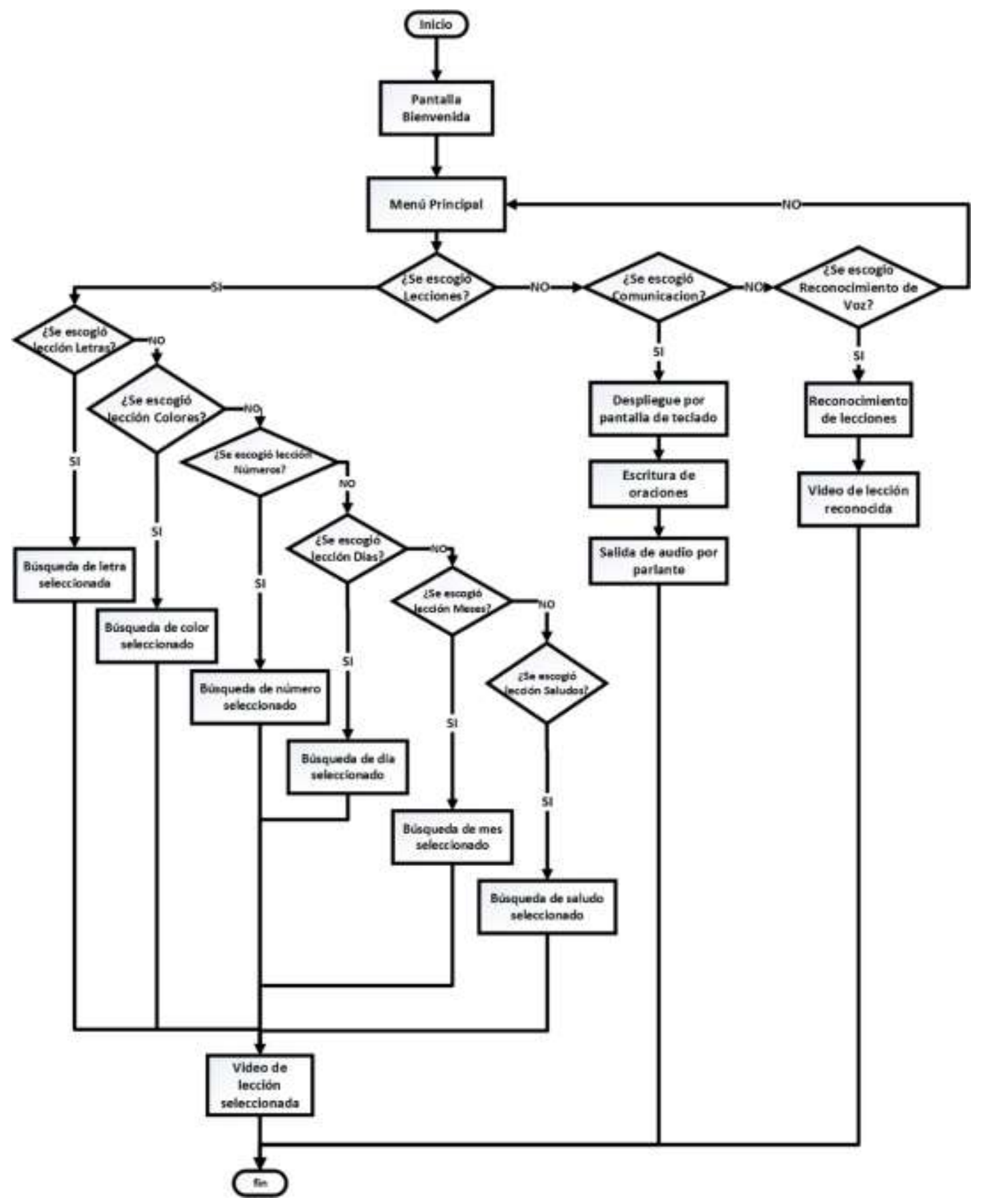

Fig. 2: Diagrama de Navegación por los menús del prototipo

Cuando el usuario selecciona el menú reconocimiento de voz, el prototipo muestra una nueva pantalla con las teclas iniciar y salir. El proceso especifico del reconocimiento de voz se explicara en detalla en el modulo de Reconocimiento de Voz.

\section{Módulo de Visualización}

Este módulo hace uso de la pantalla grafica táctil dispuesta en el prototipo y se centra en el menú Lecciones en donde se encuentra la mayor interactividad del usuario y el sistema.

De acuerdo a la lección seleccionada, el sistema mostrara los ítems incluidos en la lección. Si por ejemplo la lección escogida es "Letras", el sistema mostrara las Letras dentro de un recuadro cada una de ellas, a fin de ser seleccionadas. Cuando el usuario presiona con el dedo el recuadro que representa una letra, el sistema mostrara un video donde se describe la seña que representa la letra escogida. Cabe anotar que cualquiera de las lecciones escogidas tendrá a su vez ítems y que cada uno de estos tiene un video asociado donde se hace la representación del mismo en lengua de señas, como lo muestra la Fig. 3 para el caso del menú lecciones. 


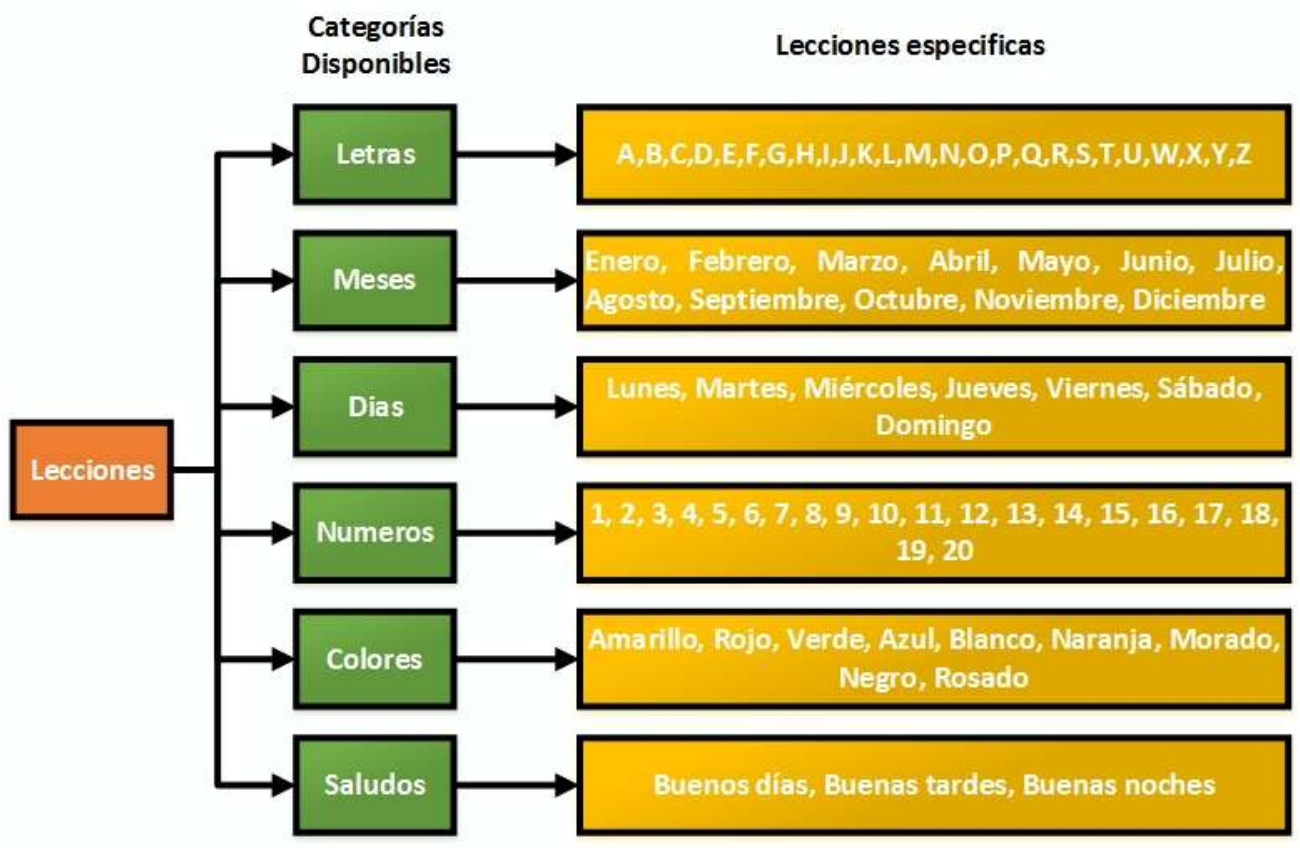

Fig. 3: Opciones de lección y lecciones

\section{Módulo de Síntesis de Voz}

Consiste en la conversión a voz de un texto introducido por el usuario mediante el teclado virtual generado en la pantalla, de forma que el usuario pueda expresar sus ideas $y / 0$ inquietudes a las personas sin discapacidad auditiva. Este módulo se compone del sintetizador de Voz DOUBLETALK RC8660FP de RC Systems (2010) y un parlante de impedancia de $8 \Omega$ a 2W. Este módulo se comunica a través de comunicación serial con la pantalla, desde la cual recibe la información que se desea convertir a voz.

\section{Módulo de Reconocimiento}

Consiste en el reconocimiento de un conjunto de palabras previamente determinadas, las cuales una vez pronunciadas son reconocidas por el dispositivo y mostradas en la pantalla grafica táctil a través del video en lengua de señas correspondiente. El reconocimiento de voz utilizado es independiente del habla, lo que significa que las palabras podrán ser pronunciadas por cualquier persona sin necesidad de realizar un entrenamiento previo. Cuando el usuario selecciona el menú reconocimiento de voz, el prototipo muestra una nueva pantalla con los botones "iniciar" y "salir" Una vez que el usuario presiona el botón "Iniciar", la directiva es enviada al microcontrolador del prototipo, el cual se encarga de iniciar de manera adecuada el módulo de reconocimiento y una vez que se encuentra listo para reconocer, se lo hace saber al usuario encendiendo un led verde. Una vez el led verde se enciende, la persona puede mencionar una de las palabras previamente establecidas, si la palabra es reconocida, el modulo enviará dicha palabra para que sea visualizada, en caso contrario el módulo de reconocimiento informara que la palabra no ha sido reconocida.

\section{Módulo de Carga de Batería}

Este módulo se encarga de realizar la carga de la batería disponible en el prototipo cuando ésta se descargue parcial o completamente, para lo cual cuenta con un sistema de monitorización constante que permite medir el nivel de carga restante de la batería y visualizar a través de un led rojo cuando este sea bajo. El proceso de carga se realiza de forma lenta para extender la vida útil de la misma, por lo que el tiempo de carga es aproximadamente de seis horas. El módulo de carga de batería se compone de tres etapas: etapa de alimentación externa, etapa de control de carga de batería y etapa de regulación y filtrado de voltaje, las cuales se explican a continuación: i) La etapa de alimentación externa se compone del conector de energía disponible en el prototipo, donde se acopla el adaptador de energía para suministrar el voltaje de carga de la batería; ii) La etapa de control de carga de batería se compone por un circuito que controla la corriente de carga, manteniendo un led amarillo encendido el cual se apaga una vez la batería ha sido cargada completamente; iii) La etapa de regulación y filtrado de voltaje se compone de dos reguladores de $5 \mathrm{~V}$ y $3.3 \mathrm{~V}$ con sus respectivas configuraciones de filtrado, para estabilizar el voltaje. La razón por la cual se utilizan dos reguladores de voltaje es debido a que la pantalla solo funciona a $5 \mathrm{~V}$ mientras que los otros 
dispositivos tales como el sintetizador, microcontrolador y modulo reconocimiento solo pueden trabajar de manera segura a $3.3 \mathrm{~V}$; y iv) El módulo de carga también cuenta con un sistema de ahorro de energía que se extiende a al sintetizador de voz, al reconocimiento de voz y a la pantalla grafica táctil. El sistema de ahorro de energía se pone en funcionamiento cuando el tiempo de inactividad excede los 10 segundos, haciendo que los dispositivos mencionados entren en modo de reposo, para el caso de la pantalla grafica táctil inicialmente se reducirá su nivel de brillo y posteriormente se apagará.

\section{RESULTADOS}

El principal resultado de esta investigación fue el desarrollo del dispositivo funcional, cuyo aspecto final se muestra en la Fig. 4.

Los resultados alcanzados en el desarrollo del presente proyecto se clasificaron en resultados técnicos y resultados de validación. Los primeros se obtuvieron a través de pruebas realizadas en laboratorio y están resumidos en las tablas 1 a la 5, los segundos se obtuvieron a través de experimentos y pruebas realizadas con una muestra de la población objeto de esta investigación.

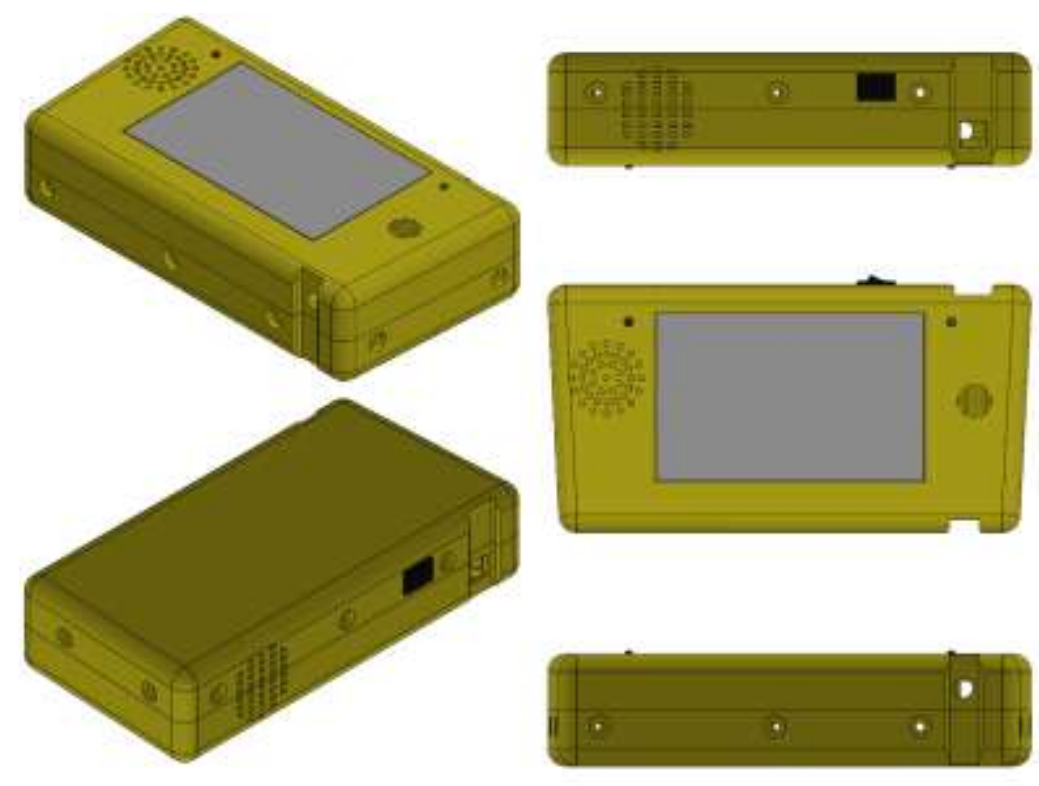

Fig. 4: Vistas del dispositivo

\section{Evaluación Técnica}

Las especificaciones técnicas del prototipo tales como voltaje, amperaje y tamaño, se describen en la Tabla 1. La Tabla 2 describe los parámetros de consumo de cada uno de los componentes del dispositivo en estado de reposo, cargando, apagado y a plena carga. La Tabla 3 describe el tiempo de autonomía del dispositivo, es decir durante cuánto tiempo puede funcionar con la carga de la batería completa.

Tabla 1: Especificaciones Técnicas del Prototipo

\begin{tabular}{|l|l|}
\hline Características & Medidas \\
\hline Fuente Alimentación & $9 \mathrm{VDC}$ \\
\hline Voltaje Batería & $7,4 \mathrm{~V}$ \\
\hline Corriente Batería & $0.45 \mathrm{~A}$ \\
\hline Señalización & $3 \mathrm{Leds}$ \\
\hline Corriente consumida por el dispositivo Usando amplificador de audio & $401 \mathrm{~mA}$ \\
\hline Pantalla Táctil & $3.3 \mathrm{Pulg}$ \\
\hline Altura del dispositivo & $9.3 \mathrm{~cm}$ \\
\hline Ancho del dispositivo & $11.6 \mathrm{~cm}$ \\
\hline Profundidad del dispositivo & $4 \mathrm{~cm}$ \\
\hline Volumen & $431.52 \mathrm{~cm}^{3}$ \\
\hline Consumo (Pleno Uso) & $1.5868 \mathrm{~W} / \mathrm{h}$ \\
\hline Peso & $450 \mathrm{~g}$ \\
\hline
\end{tabular}


Tabla 2: Consumo del dispositivo

\begin{tabular}{|c|c|c|c|c|}
\hline Parámetros & Estado Reposo & Estado Apagado & $\begin{array}{l}\text { Estado Cargando } \\
\text { (Disp Encendido) }\end{array}$ & En pleno Uso \\
\hline Voltaje & $\begin{array}{l}\text { 3.3 V:Sintetizador de } \\
\text { voz } \\
\text { 3.3 V:Modulo } \\
\text { Reconocimiento } \\
\text { 5V:Pantalla Grafica }\end{array}$ & $\begin{array}{l}0 \mathrm{~V} \text { : Sintetizador de } \\
\text { voz } \\
\text { OV:Modulo } \\
\text { Reconocimiento } \\
\text { OV:Pantalla Grafica }\end{array}$ & $\begin{array}{l}\text { 3.3 V:Sintetizador de voz } \\
\text { 3.3V: Modulo } \\
\text { Reconocimiento } \\
\text { 5V:Pantalla Grafica }\end{array}$ & $\begin{array}{l}\text { 3.3 V:Sintetizador de } \\
\text { voz } \\
\text { 3.3V:Modulo } \\
\text { Reconocimiento } \\
\text { 5V:Pantalla Grafica }\end{array}$ \\
\hline Corriente & $\begin{array}{l}1 \mathrm{~mA} \text { : Modulo } \\
\text { Reconocimiento } \\
4 \mathrm{~mA} \text { : Pantalla } \\
\text { Grafica } \\
\text { 700uA:Sintesis de } \\
\text { voz }\end{array}$ & $\begin{array}{l}0 \mathrm{~mA} \text { : Modulo } \\
\text { Reconocimiento } \\
0 \mathrm{~mA}: \text { Pantalla } \\
\text { Grafica } \\
0 \mathrm{~mA}: \text { Sintesis de voz }\end{array}$ & $\begin{array}{l}26 \text { mA: Modulo } \\
\text { Reconocimiento } \\
155 \text { mA: Pantalla Grafica } \\
220 \mathrm{~mA}: \text { Sintesis de voz } \\
\text { (usando amplificador) }\end{array}$ & $\begin{array}{l}26 \mathrm{~mA} \text { : Modulo } \\
\text { Reconocimiento } \\
155 \mathrm{~mA}: \text { Pantalla } \\
\text { Grafica } \\
220 \mathrm{~mA} \text { : Sintesis de } \\
\text { voz (Usando } \\
\text { amplificador) }\end{array}$ \\
\hline Potencia & $0.02561 \mathrm{~W}$ & $0 \mathrm{~W}$ & $1.5868 \mathrm{~W}$ & $1.5868 \mathrm{~W}$ \\
\hline
\end{tabular}

Tabla 3: Autonomía de la batería del dispositivo

\begin{tabular}{|l|l|l|l|l|}
\hline Parámetros & $\begin{array}{l}\text { Estado } \\
\text { Reposo }\end{array}$ & Estado Apagado & $\begin{array}{l}\text { Estado Cargando (Dispositivo } \\
\text { Encendido) }\end{array}$ & En pleno Uso \\
\hline Autonomía & 230 minutos & 900 minutos & 200 minutos & 130 minutos \\
\hline
\end{tabular}

La Tabla 4 muestra los tiempos de retardo cuando se navega por las diferentes aplicaciones del dispositivo desarrollado. Finalmente la Tabla 5 muestra los costos de cada uno de los componentes del dispositivo desarrollado y el costo marginal total del mismo.

Tabla 4: Retardos entre las diferentes pantallas mostradas en el dispositivo

\begin{tabular}{|l|c|}
\hline Parámetro & Unidad \\
\hline Retardo en Encendido & $1.25 \mathrm{seg}$ \\
\hline Retardos entre Pantallas & $0.83 \mathrm{seg}$ \\
\hline Retardo entre Mensaje de Bienvenida y Menú Principal & $1.86 \mathrm{seg}$ \\
\hline Retardo en Carga de Videos & $1.23 \mathrm{seg}$ \\
\hline Probabilidad de no respuesta a comando táctil & $8 \%$ \\
\hline
\end{tabular}

Tabla 5: Costo marginal del Dispositivo

\begin{tabular}{|l|r|r|}
\hline \multicolumn{3}{|c|}{ Costo Marginal del Dispositivo } \\
\hline Componente & Cantidad & Precio Aprox \\
\hline Pantalla LCD 3.2" & 1 & $\$ 150,750.00$ \\
\hline Impreso & 1 & $\$ 22,000.00$ \\
\hline Sintetizador de voz & 1 & $\$ 130,000.00$ \\
\hline Modulo Reconocimiento & 1 & $\$ 283,100.00$ \\
\hline Diseño y construcción de la caja & 1 & $\$ 140,000.00$ \\
\hline Componentes Discretos de la Tarjeta & 1 & $\$ 28,000.00$ \\
\hline Batería & 1 & $\$ 18,000.00$ \\
\hline
\end{tabular}




\section{Validación}

Para realizar el proceso de validación se diseñó una encuesta que se practicó a un universo de 24 personas comprendidas por 12 niños oyentes, 8 niños sordos y 4 profesionales del área, con interés en el aprendizaje del lenguaje a señas, del colegio distrital San Francisco en la ciudad de Bogotá. La encuesta se conformó de 24 preguntas, 19 de ellas de carácter cuantitativo y las restantes 5 de carácter cualitativo, con el objetivo de evaluar los siguientes aspectos: dispositivo como herramienta efectiva en el aprendizaje, tiempos de aprendizaje, motivación en el uso del dispositivo y calidad en el funcionamiento del dispositivo. Con base en las anteriores áreas, se describen los principales resultados obtenidos por la encuesta.

Ante la pregunta icree usted que el prototipo mostrado permite mejorar los tiempos de aprendizaje de lenguaje a señas? El 100\% de los encuestados manifestó que si permite tener una mayor apropiación de la lengua de señas, a través de la repetición continua de los videos correspondientes.

Ante la pregunta ¿el prototipo muestra de manera clara la representación en lengua de señas de cada una de las lecciones? El $100 \%$ de los encuestados confirmaron que cada uno de los videos disponibles en las lecciones mostradas en el prototipo representa de una manera clara la lengua de señas. Incluso algunos de los encuestados justificaron que el prototipo muestra de una manera divertida y "chévere" la representación de la lengua de señas.

Ante la pregunta ¿cómo calificaría la calidad del prototipo mostrado enfocado a ser una herramienta de ayuda en el aprendizaje donde 10 representa alta calidad y 1 representa baja calidad? Los resultados fueron clasificados en Calidad Alta para el rango de 10 a 8, Calidad Media para el rango de 7 a 5 y Calidad Baja el rango de 4 a 1, y se pueden observar en la Fig. 5. Las personas que calificaron el dispositivo en calidad baja y media, argumentaron que la reproducción del texto no era muy clara en algunas ocasiones, y que en otras era necesario repetir dos veces determinada palabra para que el dispositivo la reconociese. Lo primero se solucionó a partir de un ajuste al circuito de amplificación de audio, y lo segundo se debió a la falta de vocalización correcta por parte de los niños normo-oyentes. La discriminación de los resultados por grupos de participantes se presenta en la Tabla 6.

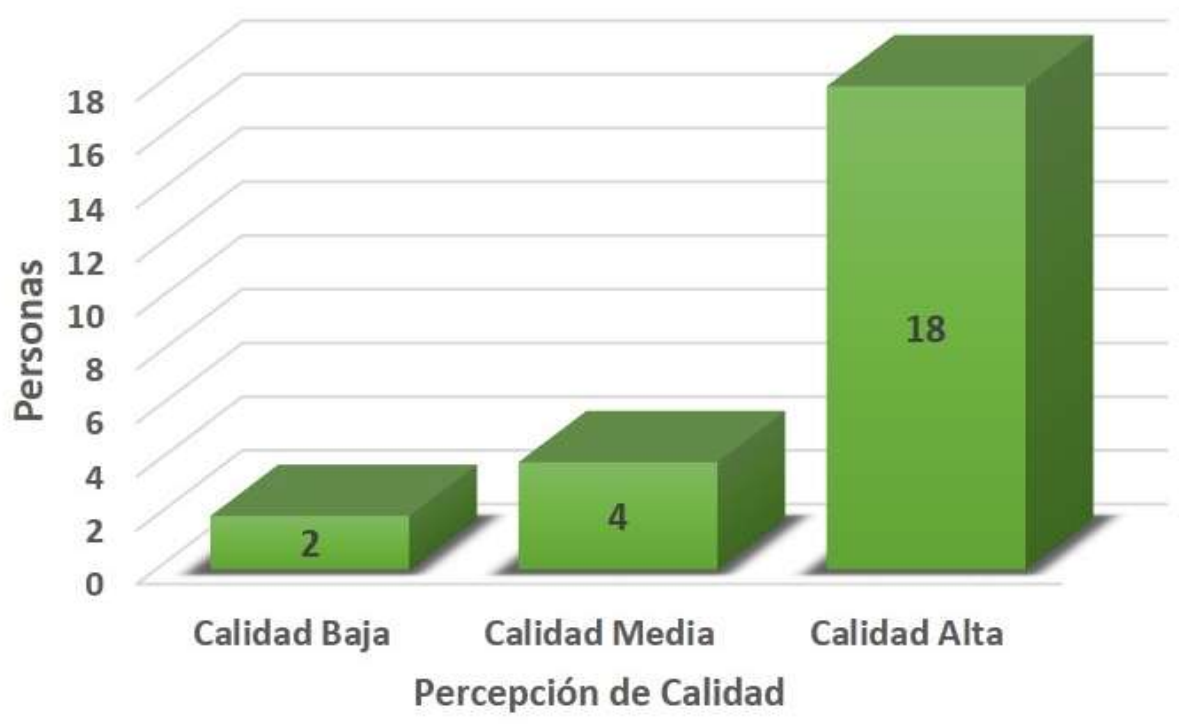

Fig. 5: Resumen de la calidad del prototipo en términos de calidad Alta, Media o Baja

Tabla 6: Distribución de los resultados de la figura 5 de acuerdo con el tipo de participante

\begin{tabular}{|l|c|c|c|c|}
\hline \multicolumn{1}{|c|}{ Tipo de Participante } & Calidad Baja & Calidad Media & Calidad Alta & Total \\
\hline Niños oyentes & 2 & 2 & 8 & 12 \\
\hline Niños sordos & 0 & 1 & 7 & 8 \\
\hline Profesionales del área & 0 & 1 & 3 & 4 \\
\hline Total & 2 & 4 & 18 & 24 \\
\hline
\end{tabular}


Ante la pregunta ¿la interfaz gráfica mostrada por el prototipo le parece fácil de manejar? Los resultados obtenidos evidencian que el $92 \%$ de los encuestados coincidió en afirmar que la interfaz gráfica era fácil de manejar, puesto que utiliza una pantalla grafica táctil similar a una Tablet. Sin embargo, el $8 \%$ de los encuestados no tienen la percepción de que sea fácil de manejar, puesto que en algunas ocasiones (inferiores al $10 \%$ de las veces) al presionar la pantalla, ésta no respondía a la acción solicitada.

Ante la pregunta ¿cuántas veces debe repetir el video antes de aprender correctamente una seña? Se obtuvieron los resultados mostrados en la Fig. 6 , donde el $50 \%$ de los encuestados afirman que deben repetir el video 102 veces para aprender una sola seña, el 17\% afirma que para ellos este número es 3 , mientras que el $8 \%$ dice necesitar repetir el video entre 4 y 12 veces. Las personas que contestaron 12 , argumentaron que tuvieron problemas para manipular el dispositivo debido a que casi nunca tienen acceso a este tipo de tecnología, y las personas que contestaron N/A no recordaron cuantas veces tuvieron que repetir el video. La discriminación de los resultados por grupos de participantes se presenta en la Tabla 7.

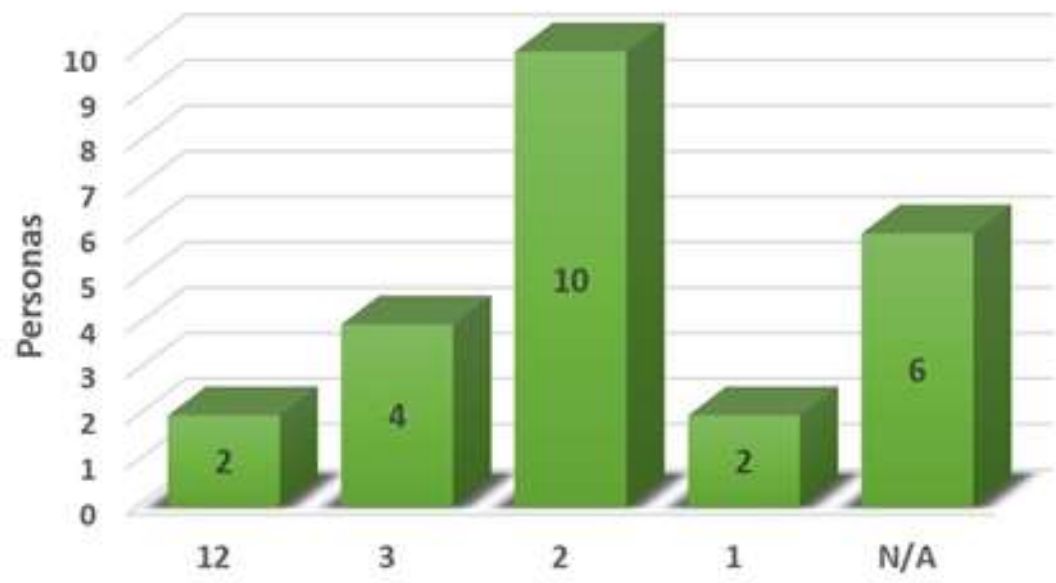

Fig. 6: Número de veces que debe repetir el video antes de aprender correctamente una seña

Tabla 7: Distribución de los resultados de la figura 6 de acuerdo con el tipo de participante

\begin{tabular}{|l|c|c|c|c|c|}
\hline \multicolumn{1}{|c|}{ Tipo de Participante } & $1-2$ veces & 3 veces & $4-12$ veces & N/A & Total \\
\hline Niños oyentes & 6 & 3 & 1 & 2 & 12 \\
\hline Niños sordos & 2 & 1 & 1 & 4 & 8 \\
\hline Profesionales del área & 4 & 0 & 0 & 0 & 4 \\
\hline Total & 12 & 4 & 2 & 6 & 24 \\
\hline
\end{tabular}

Ante la pregunta ¿Cómo califica la calidad de los videos del dispositivo, alta, media o baja? Se obtuvieron los resultados mostrados en la Fig. 7 , donde el $59 \%$ de los encuestados afirman que la calidad de los videos es alta, $8 \%$ media, $8 \%$ baja y $25 \%$ no respondió.

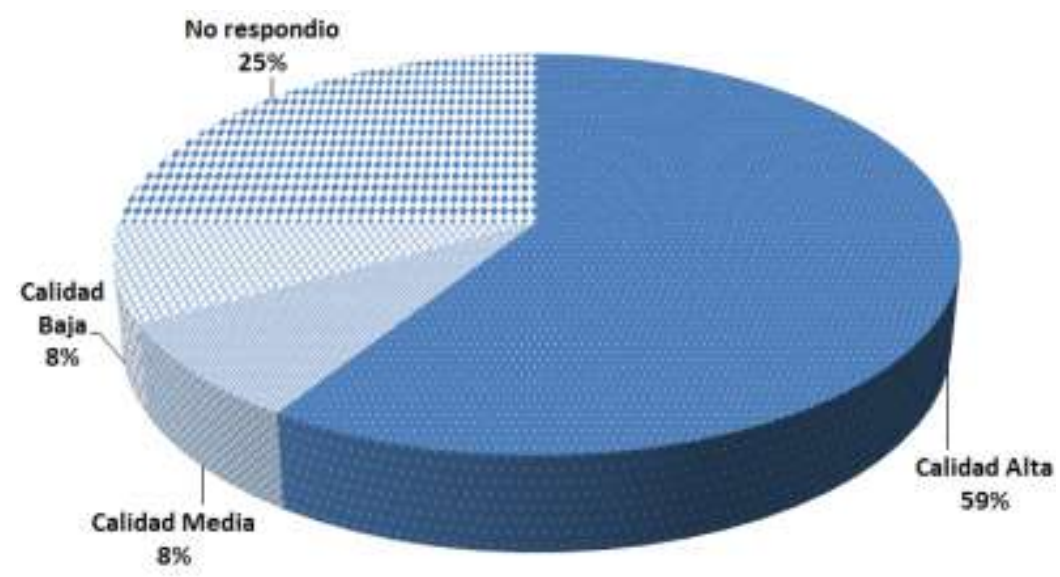

Fig. 7: Apreciación de la calidad de los videos del dispositivo 
Se realizó un test para medir el desempeño del aprendizaje de la lengua de señas para determinar cuánto tiempo le toma al niño sordo, perfeccionar una seña sencilla y una complicada con el dispositivo y sin él. De los resultados se observó que a pesar de ser una seña sencilla o complicada, los tiempos no varían significativamente. Para el $62 \%$ la demora es de 2 a 3 minutos con el dispositivo y de 3 a 5 sin él, para el $38 \%$ restante los tiempos son similares con y sin el dispositivo y oscilan entre 8 y 10 minutos. A través de un taller que se realizó en dos sesiones de cuatro horas cada una, los grupos de niños sordos y normo-oyentes interactuaron con el dispositivo para alcanzar el objetivo de aprender un grupo de señas determinado inicialmente por el docente de la clase, durante un periodo de dos horas. Las dos horas restantes de cada sesión se asignaron para que los niños interactuaran libremente con el dispositivo. Durante estas últimas dos horas se evidenció un fuerte interés por parte de los niños de aprender más signos de la lengua de señas e interactuar con sus compañeros normo-oyentes a través del dispositivo, el cual gracias a su característica de portabilidad les permitió desplazarse con el dispositivo por todo el salón e interactuar a través de el con sus compañeros.

\section{DISCUSION}

La herramienta tecnológica propuesta integra las tecnologías de la información y las comunicaciones anteriormente descritas para reforzar el sistema de aprendizaje de los niños sordos y sus familiares o amigos cercanos, de forma que el usuario ya sea de manera autónoma o con acompañamiento, pueda mejorar sus tiempos de aprendizaje.

De acuerdo con los resultados de las encuestas el dispositivo desarrollado se perfila como una herramienta robusta y complementaria en el proceso de aprendizaje de la lengua de señas, tanto en personas normooyentes como las que presentan algún tipo de discapacidad auditiva. Las características de portabilidad e interactividad del dispositivo permiten, facilitan y fortalecen el aprendizaje autónomo de la lengua de señas, el cual en la enseñanza tradicional difícilmente se da.

Con la realización de los talleres y test se pudo comprobar el aporte que hace el dispositivo a la motivación del estudiante por aprender la lengua de señas y a su deseo de interactuar con sus compañeros normooyentes. Lo anterior repercutió directamente en la reducción de los tiempos promedio de aprendizaje de los signos de la lengua de señas, entre $20 \%$ y $40 \%$.

A pesar de que el dispositivo desarrollado tiene por objetivo principal facilitar el aprendizaje de la lengua de señas, también está diseñado para convertirse en un intérprete bidireccional entre las personas sordas y normo-oyentes. Los resultados descritos anteriormente muestran un gran porcentaje de aceptación de las funciones de síntesis y reconocimiento de voz, las cuales permiten dicha interacción. Lo anterior hace una gran diferencia con los trabajos relacionados en la introducción, ya que casi todos ellos se enfocan en un solo tema y no son muy versátiles a diferencia de la herramienta tecnológica propuesta.

\section{CONCLUSIONES}

El sistema desarrollado es una herramienta complementaria en el proceso de aprendizaje del lenguaje de señas y refuerza este proceso, generando autonomía en el estudio por parte de los usuarios del dispositivo, logrando una sinergia entre la parte pedagógica y la tecnología. El proyecto realizado genera un aporte que apunta a ser una solución relevante en la problemática de inclusión social a la que están expuestas las personas con discapacidad auditiva en Colombia. El dispositivo tecnológico realizado puede ser usado tanto por personas sordas como por personas oyentes. En el caso de las personas sordas el dispositivo constituye una herramienta de aprendizaje básico y una posibilidad de comunicación haciendo uso de la función de voz, en tanto que para las personas oyentes constituye una herramienta didáctica para el aprendizaje del lenguaje de señas, para poder comunicarse con la persona sorda, ayudando a reducir la brecha de inclusión.

\section{AGRADECIMIENTOS}

El presente artículo es producto de los resultados alcanzados en el proyecto de investigación "Dispositivo de comunicación para personas sordomudas", financiado por la Universidad Distrital Francisco José de Caldas y Colciencias, a través del programa "Jóvenes Investigadores e Innovadores". También queremos agradecer a todas las personas del colegio San Francisco, de la localidad de Ciudad Bolívar en la ciudad de Bogotá, que nos colaboraron durante todo el proceso de validación del dispositivo.

\section{REFERENCIAS}

Alonso, A. y Díaz, E; Universidad y discapacidad: indicadores de buenas prácticas y estándares de actuación para programas y servicios. SIGLOCERO Rev. .Esp.Discapacidad Intelectual, 39(2), 82-98 (2008) 
Chaves, E., Trujillo, J.M. y López, J.A.; Autorregulación del Aprendizaje en Entornos Personales de Aprendizaje en el Grado de Educación Primaria de la Universidad de Granada, España, doi: 10.4067/S0718-50062015000400008, Form. Univ. (en línea), 8(4), 63-76 (2015)

Chiroleu, A; La educación superior en américa latina: ¿problemas insolubles o recetas inadecuadas? Avaliação (Campinas), 16(3), 631-653 (2011)

Domínguez, A. B. y Rodríguez, P.; Se puede enseñar conocimiento fonológico a los niños sordos? Is it possible to teach phonological awareness to deaf children? Infancia y aprendizaje, 26(4), 485-501 (2003)

Escalante, J.; Enfrentando los nuevos retos educativos. Una mirada profunda a los entornos educativos, mediante un análisis sistemático y consecuente, dando como resultado propuestas fundamentadas en la solución de las problemáticas educativas actuales, Revista Educación hoy, 1-12 (2012)

Espinosa, Carlos X, Gómez, Víctor G, y Cañedo, C.M.; El Acceso y la Retención en la Educación Superior de Estudiantes con Discapacidad en Ecuador, doi: 10.4067/S0718-50062012000600004, Form. Univ. (en línea), 5(6), 27-38 (2012)

Fuller, M., Bradley, A. y Healey, M.,; Incorporating disabled students within an inclusive higher education environment, Disability and Society, 19, 455-468 (2004)

Gwendolyn, S. Method of providing two-way communication between a deaf person and a hearing person. International Patent US20110116608A1, 18 Nov. (2009), App.12/621,097, 19 May (2011)

Hao, Z., Analogue circuit teaching demonstration board for deaf students. International Patent CN201845462, 25 May (2011), CN 201020509238, 30 Aug. (2010)

Hernández, C. y Márquez H., Dispositivo electrónico portátil que facilita el aprendizaje inicial del lenguaje de señas en niños con discapacidad auditiva y del habla, Colombia, 13-244024, G06F 3/01 Octubre (2013)

Hernández, C., Pulido, J.L, y Arias, J.E., Las tecnologías de la información en el aprendizaje de la lengua de señas, doi: 10.15446/rsap.v17n1.36935, Rev. Salud Pública (en línea), 17(1), 61-73 (2015)

Hernández, M.R, Rodríguez, V.M, Parra, F.J., y Velázquez, P.; Las Tecnologías de la Información y la Comunicación (TICs) en la Enseñanza-Aprendizaje de la Química Orgánica a Través de Imágenes, Juegos y Video, doi: 10.4067/S0718-50062014000100005, Form. Univ. (en línea), 7(1), 31-40 (2014)

Hernández-Sánchez, A.M., y Ortega, J.A.; Aprendizaje Electrónico Afectivo: un modelo Innovador para Desarrollar una Acción Tutorial Virtual de Naturaleza Inclusiva, doi: 10.4067/S0718-50062015000200004, Form. Univ. (en línea), 8(2), 19-26 (2015)

Instituto Nacional para Sordos, Estadísticas e información para contribuir en el mejoramiento de la calidad de vida de la población sorda colombiana, (en la web

http://www.insor.gov.co/historico/images/bolet\%C3\%ADn\%20observatorio.pdf, acceso: Junio de 2013), INSOR (2009)

Instituto Nacional para Sordos, Lenguaje a Señas (en la web http://www.insor.gov.co acceso: Julio 2013), INSOR (2013)

Jockey, P., Stuffed Bear as teaching aid. U.S Patent 4799889, 24 Jan. (1989)

Kramer, J. P. Lindener, P. y George, W. R., Communication system for deaf, deaf-blind, or non-vocal individuals using instrumented glove U.S. Patent 5047952, 10 Sept. (1991), US 07/258,204, 14 Oct. (1988)

Laenger, C. J. McFarland, S. R. y Peel, H. H., Method and apparatus for communicating with people U.S. Patent 4074444, 21 Feb. (1978), US 05/728,404, 30 Sept. (1976)

Marulanda, C.E, Giraldo, J., y López, M., Acceso y Uso de las Tecnologías de la Información y las Comunicaciones (TICS) en el Aprendizaje: El Caso de los Jóvenes Preuniversitarios en Caldas, Colombia, doi: 10.4067/S0718-50062014000400006, Form. Univ. (en linea), 7(4), 47-56 (2014)

Masfrand, O. D. F., M. Word constructionless communication method for dumb and/or deaf, involves 
displaying detail or photo or pictogram on screen and/or vocalizing message using synthesis or recording voice. International Patent FR2960999, 9 Dec. (2011), FR20100002412 20100608, Jun. (2010)

RC Systems, Sintetizador de Voz, (en la web http://www.rcsys.com/Downloads/rc8660.pdf ${ }_{2}$ acceso: Junio de 2013). RC Systems (2011)

Rudner M., Andin J., Rönnberg J., Heimann M., Hermansson A., Nelson K. y Tjus T.; Training Literacy Skills through Sign Language. Deafness \& Education International, 17(1), 8-18 (2015)

Salvador, F. y Gutiérrez, R., El proceso de planificación en la expresión escrita de alumnos sordos: estudio de casos en educación secundaria, Revista de educación, 339, 435-453 (2006)

Sapora, R., Educational apparatus and method for control of deaf individuals in a mixed teaching environment. U.S. Patent 4368459, 11 Jan. (1983), US 06/217,081, 16 Dec. (1980)

Skliar, C., La educación de los sordos. Una reconstrucción histórica, cognitiva y pedagógica, Mendoza: EDIUNC, (1997)

Sparks, W. B., Educational device especially for the blind and the blind-deaf. U.S. Patent 4378215. 29 Mar. 1983, US 06/272,514, 11 Jun. (1981)

Swanwick R., Policy and practice in sign bilingual education: development, challenges and directions, doi: 10.1080/13670050903474069, International Journal of Bilingual Education and Bilingualism (en línea), 13(2) 147-158 (2010)

Tamarit, J., Los trastornos de la comunicación en deficiencia mental y otras alteraciones evolutivas: intervención mediante Sistemas de Comunicación Total en Comunicación aumentativa, Madrid: INSERSO, 137-162 (1988)

Tur, C. y Fernández, M. P.; La mediación semiótica en el sordo: Algunas reflexiones, Revista de Logopedia, Foniatría y Audiología, 12(4), 213, (1992)

Yang, H. y Zhang, J., Chatting machine for deaf mute. International Patent CN201984680, 25 Mar. (2011), CN 201120082488, 21 Sept. (2011) 